\title{
Spinal subarachnoid hematoma after spinal anesthesia
}

\author{
Sung Bae Jeon, Tae-In Ham, Min-Su Kang, Ho-Yong Shim, and Sang Lee Park \\ Department of Anesthesiology and Pain Medicine, National Police Hospital, Seoul, Korea
}

Moen et al. [1] reported that spinal hematoma occurred in 8 out of 1,260,000 cases of spinal anesthesia in Sweden, and subarachnoid hematoma is known to be very rare [2]. We report a case of subarachnoid hematoma after spinal anesthesia in a patient without coagulopathy.

A 33-year-old man with a height of $170 \mathrm{~cm}$ and weight of 65 $\mathrm{kg}$ underwent emergency surgery for acute appendicitis without perforation. The patient had no specific medical history and was taking no medications. Preoperative laboratory examinations including platelet count $\left(236,000 / \mathrm{mm}^{3}\right)$, prothrombin time (12.2 seconds, with international normalized ratio 1.07), and activated partial thromboplastin time (29.5 seconds) were in the normal range.

The patient was placed in the right lateral decubitus position, and spinal anesthesia was attempted at the L4-5 intervertebral space via a midline approach using a $25 \mathrm{G}$ Quincke spinal needle (Hakko Co., Naganoken, Japan). On the first trial, the needle reached the spinous process before it passed the ligamentum flavum, so it was removed immediately. On the second trial, clear cerebrospinal fluid without blood was observed without any particular difficulties. Fourteen milligrams of $0.5 \%$ hyperbaric bupivacaine mixed with fentanyl $15 \mu \mathrm{g}$ was injected without pain, numbness, or dysesthesia. The patient was placed in the supine position, and the level of sensory block was $\mathrm{T} 7$ at 5 minutes after intrathecal injection. The operation was uneventful, and he was discharged without any specific neurologic symptoms. Five days after the spinal anesthesia, the patient called complaining of lower back pain and numbness in the lower limbs without allodynia, hypoesthesia, or paralysis. The complications that can occur after spinal anesthesia, such as transient neurologic symptoms (TNS), hematoma, abscess, cauda equina syndrome, spinal neurological damage, muscle rigidity, and myofascial pain, were explained to the patient, and he was asked to come to the hospital. However, the patient wanted to go to another hospital because of his personal affairs. Magnetic resonance imaging performed at the other hospital showed the presence of a subarachnoid hematoma (Fig. 1). The patient was mornitored closely but received no other treatment because the lesion was not large and his symptoms were improving. Ten days after the spinal anesthesia, his symptoms had all resolved.

Spinal subarachnoid hematoma cases reported up to now have occurred mostly in patients who had problems with blood coagulability or in instances when anticoagulant was admini-

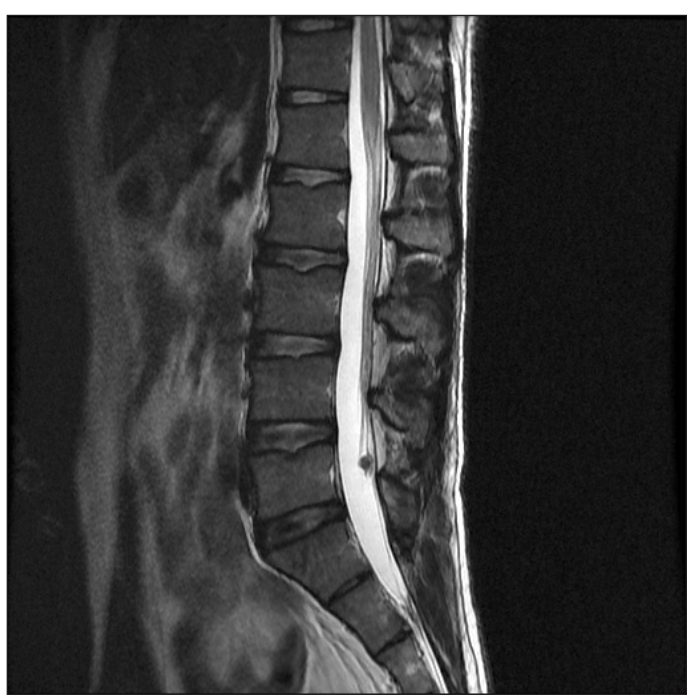

Fig. 1. T2-weighted sagittal magnetic resonance imaging shows a subarachnoid hematoma near L5. The hypo-intense signal in the T2weighted image is consistent with deoxyhemoglobin.

Corresponding author: Sang Lee Park, M.D., Department of Anesthesiology and Pain Medicine, National Police Hospital, Garakbon-dong, Songpa-gu, Seoul 138-708, Korea. Tel: 82-2-3400-1325, Fax: 82-2-3400-1322, E-mail: slpak@nph.go.kr

(c) This is an open-access article distributed under the terms of the Creative Commons Attribution Non-Commercial License (http:// creativecommons.org/licenses/by-nc/3.0/), which permits unrestricted non-commercial use, distribution, and reproduction in any medium, provided the original work is properly cited. 
stered. Recently, Karakosta et al. [3] reported a subarachnoid hematoma occurring after spinal anesthesia in a 74-year-old male patient without any known blood coagulability disorder. In Comparison, the case we report here is unusual in that subarachnoid hematoma is very uncommon in patients as young as 33 years old.

Subarachnoid hematoma is very rare even among spinal canal hematomas. The cause of this rarity is thought to be that it is difficult to generate a hematoma even when bleeding occurs, because the blood is diluted with cerebrospinal fluid and flushed with the flow of the fluid. Therefore, it is thought that spondylosis, herniation of intervertebral discs, arachnoiditis, and ligamentum flavum hypertrophy that can disturb the flow of spinal fluid may contribute to the formation of hematomas [4]. On the other hand, even when there are no disorders in the spinal canal, hematoma can occur if there is sufficient bleeding. In practice, the major contributing factor to bleeding or hematoma formation after spinal anesthesia is thought to be a problem in blood coagulability. Out of the 31 subarachnoid hematoma cases occurring after spinal anesthesia analyzed by Domenicucci et al. [4], a blood coagulability disorder was present in 23 cases $(74 \%)$. In many of these cases, the blood coagulability disorder was caused by the use of an anticoagulant. In addition, the use of anticoagulant may cause a hematoma even when blood coagulability test results are normal [5].

In many cases of failed spinal anesthesia due to lack of cerebrospinal fluid, subarachnoid hematomas have been reported. Therefore, regardless of cerebrospinal fluid, all spinal puncture trials should be specified [5]. Minor symptoms of small subarachnoid hematomas can be confused with TNS [5]. The conspicuous difference between the two for differential diagnosis is the duration of the symptoms. Most symptoms of TNS disappear within two days. In the case reported here, however, the pains of spinal hematoma were persistent until 5 days after spinal anesthesia.

MRI is the most useful test to diagnose subarachnoid hematoma [2]. However, if the hematoma is closely attached to the arachnoid, especially when the hematoma is located in front of the spinal cord, it is difficult to distinguish between a hematoma, arachnoid, and dura mater, and consequently it is difficult to distinguish subarachnoid hematoma from subdural hematoma. In such cases, a definitive diagnosis can only be obtained through surgical exploration. In the case discussed here, the subarachnoid hematoma could be diagnosed easily because the hematoma was detached from the arachnoid and the hematoma was surrounded by cerebrospinal fluid.

The basic treatment for subarachnoid hematoma is surgical decompression. Although the symptoms can be minor, as in our case, in most cases neurologic deficits such as sphincter disorder and lower limb paralysis are observed. In such cases, rapid surgical decompression is recommended. Recently, there have been reports of small subarachnoid hematomas with minor pains being cured by conservative treatment [3]. However, even a small hematoma should be monitored closely, as neurologic deficits might develop. In our case, the patient was observed closely and the symptoms resolved quickly.

In conclusion, when spinal anesthesia or epidural anesthesia is administered, the possibility of hematoma occurrence should always be considered regardless of the use of anticoagulant or multiple or traumatic centesis trials.

\section{References}

1. Moen V, Dahlgren N, Irestedt L. Severe neurological complications after central neuraxial blockades in Sweden 1990-1999. Anesthesiology 2004; 101: 950-9.

2. Kreppel D, Antoniadis G, Seeling W. Spinal hematoma: a literature survey with meta-analysis of 613 patients. Neurosurg Rev 2003; 26 : 1-49.

3. Karakosta A, Kyrallidou A, Chapsa C, Pouliou A. Acute spinal subarachnoid haematoma following spinal anaesthesia treated conservatively: case report. Eur J Anaesthesiol 2011; 28: 388-90.

4. Domenicucci M, Ramieri A, Paolini S, Russo N, Occhiogrosso G, Di Biasi C, et al. Spinal subarachnoid hematomas: our experience and literature review. Acta Neurochir (Wien) 2005; 147: 741-50.

5. Lam DH. Subarachnoid haematoma after spinal anaesthesia mimicking transient radicular irritation: a case report and review. Anaesthesia 2008; 63: 423-7. 